\title{
ON JOINT FUNCTIONAL CALCULUS FOR RITT OPERATORS
}

\author{
PARASAR MOHANTY AND SAMYA KUMAR RAY
}

\begin{abstract}
In this paper, we study joint functional calculus for commuting $n$-tuple of Ritt operators. We provide an equivalent characterisation of boundedness for joint functional calculus for Ritt operators on $L^{p}$-spaces, $1<p<\infty$, where each of them admits a bounded functional calculus. We also investigate joint similarity problem for commuting $n$-tuple of Ritt operators. We get our results by proving a suitable multivariable transfer principle between sectorial and Ritt operators as well as an appropriate joint dilation result in a general setting.
\end{abstract}

\section{INTRODUCTION}

Let $X$ be a Banach space and $T$ be a linear operator on $X$. In many instances it is important to assign a meaning to the symbol $f(T)$ for a bounded holomorphic function $f$ defined on an appropriate domain. From celebrated von Neumann inequality [36], we can associate bounded functional calculus for every contraction when $X$ is a Hilbert space. More precisely, if $f$ is any holomorphic function with poles outside closure of the unit disc $\overline{\mathbb{D}}$ and $T$ a contraction, then

$$
\|f(T)\| \leq\|f\|_{\infty, \mathbb{D}} \text {. }
$$

Above inequality follows for a commuting pair of contractions $\left(T_{1}, T_{2}\right)$ on a Hilbert space from a theorem by Ando [3]. However, Varopoulos [35] provided an example of the failure of this inequality for commuting 3 -tuple of contractions.

McIntosh and his coauthors [9], [24] developed a notion of $H^{\infty}$-functional calculus for sectorial operators on Banach spaces. Sectorial operators have been studied extensively. It has many applications in partial differential equations and harmonic analysis [37]. The notion of joint functional calculus for sectorial operators with commuting resolvents is due to [2], which again has important applications in studying maximal regularity problems [17], [20].

In [21] Christian Le Merdy initiated the study of functional calculus for Ritt operators. He and his coauthors in [5], [4] and [21], have obtained various results in the lines of sectorial operators and also characterised Ritt operators on $L^{p}$-spaces having bounded $H^{\infty}$-functional calculus.

In this paper, we develop a notion of joint functional calculus for commuting Ritt operators and obtain equivalent criterion for commuting tuple of Ritt operators to admit joint bounded functional calculus on $L^{p}$-space, $1<p<\infty$, where each of them also satisfies a bounded functional calculus. We apply our methods to study joint similarity problem for commuting tuple of Ritt operators acting on a Hilbert space.

\section{Notations AND MAin RESUlts}

Let $(\Omega, \mathbb{F}, \mu)$ be a $\sigma$-finite measure space and $1 \leq p \leq \infty$. We denote $L^{p}(\Omega, X)$ to be the usual Bochner space. Given any open set $D \subseteq \mathbb{C}^{n}$, we define $H^{\infty}(D)$ to be the set of all bounded holomorphic maps equipped with the supremum norm. For any set $K$ and $f: K \rightarrow \mathbb{C}$, denote $\|f\|_{\infty, K}:=\sup \{|f(z)|: z \in K\}$. If $a \in \mathbb{C}$, we denote the open disc of radius $r$ with center $a$ to be $D(a, r)$. For any set $S$ we denote $S^{n}$ by its $n$-times cartesian product and $d\left(S_{1}, S_{2}\right)$ be the usual distance between $S_{1}, S_{2} \subseteq \mathbb{C}$, i.e. $d\left(S_{1}, S_{2}\right):=\inf \left\{\left|z_{1}-z_{2}\right|: z_{1} \in S_{1}, z_{2} \in S_{2}\right\}$.

\footnotetext{
Key words and phrases. Functional Calculus, Ritt Operators, Sectorial operators .

The second author is supported by Council for Scientific and Industrial Research, MHRD, Government of India.
} 


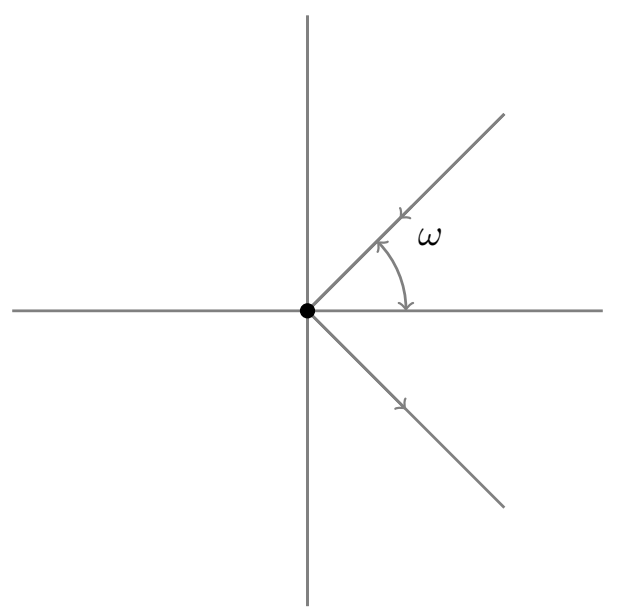

FiguRE 1.

2.1. Sectorial operators: We recall some preliminaries on sectorial operators and associated joint $H^{\infty}$-functional calculus. We recommend the interested readers [2], [11], [13], [16], [17] and [20] for more on this direction.

Definition 2.1 (Sectorial operator). For any $\omega \in(0, \pi)$, let $\Sigma_{\omega}:=\{z \in \mathbb{C} \backslash\{0\}:|\arg z|<\omega\}$ be the open sector of an angle $2 \omega$ around the positive real axis $(0, \infty)$ (see FIGURE. 1). We say that a densely defined closed operator $A: D(A) \subseteq X \rightarrow X$ with domain $D(A)$ is sectorial of type $\omega \in(0, \pi)$ if we have

(i) $\sigma(A) \subseteq \overline{\Sigma_{\omega}}$.

(ii) For any $\nu \in(\omega, \pi)$, the set $\left\{z R(z, A): z \in \mathbb{C} \backslash \overline{\Sigma_{\nu}}\right\}$ is bounded, where $R(., A)$ is the resolvent operator of $A$.

Let $\nu \in(0, \pi)$ and $\Gamma_{\nu}$ be the boundary of $\Sigma_{\nu}$, oriented counter-clockwise (see FIGURE. 1). For $\theta_{i} \in(0, \pi), 1 \leq i \leq n$, denote $H_{0}^{\infty}\left(\prod_{i=1}^{n} \Sigma_{\theta_{i}}\right)$ to be the set of all bounded holomorphic functions $f: \prod_{i=1}^{n} \Sigma_{\theta_{i}} \rightarrow \mathbb{C}$ with the property that there exists constants $C, s>0$, depending only on $f$, such that

$$
\left|f\left(z_{1}, \ldots, z_{n}\right)\right| \leq C \prod_{i=1}^{n} \frac{\left|z_{i}\right|^{s}}{1+\left|z_{i}\right|^{2 s}} \quad \text { for all }\left(z_{1}, \ldots, z_{n}\right) \in \prod_{i=1}^{n} \Sigma_{\theta_{i}} .
$$

Let $\mathbf{A}:=\left(A_{1}, \ldots, A_{n}\right)$ be an $n$-tuple of operators with mutually commuting resolvents such that each $A_{i}$ is sectorial of type $\omega_{i} \in(0, \pi), 1 \leq i \leq n$. Let $\omega_{i}<\theta_{i}<\pi$ and $\nu_{i} \in\left(\omega_{i}, \theta_{i}\right), 1 \leq i \leq n$. One defines

$$
f(\mathbf{A}):=\left(\frac{1}{2 \pi i}\right)^{n} \int_{\prod_{i=1}^{n} \Gamma_{\nu_{i}}} f\left(z_{1}, \ldots, z_{n}\right) \prod_{i=1}^{n} R\left(z_{i}, A_{i}\right) d z_{i} .
$$

The decay on the resolvent operators ensures that the integral in (1) is absolutely convergent and by Cauchy's theorem, it is independent of $\nu_{i}, 1 \leq i \leq n$. Let $\Phi_{\mathbf{A}}: H_{0}^{\infty}\left(\prod_{i=1}^{n} \Sigma_{\theta_{i}}\right) \rightarrow B(X)$ be defined as

$$
\Phi_{\mathbf{A}}(f):=f(\mathbf{A}) .
$$

Then, the map $\Phi_{\mathbf{A}}$ is an algebra homomorphism [11].

Definition 2.2. We say that $\boldsymbol{A}$ admits a joint bounded $H^{\infty}\left(\prod_{i=1}^{n} \Sigma_{\theta_{i}}\right)$ functional calculus, if for all $f \in H_{0}^{\infty}\left(\prod_{i=1}^{n} \Sigma_{\theta_{i}}\right)$, there exists a constant $C>0$ (independent of $f$ ), such that

$$
\|f(\boldsymbol{A})\|_{X \rightarrow X} \leq C\|f\|_{\infty, \prod_{i=1}^{n} \Sigma_{\theta_{i}}}
$$




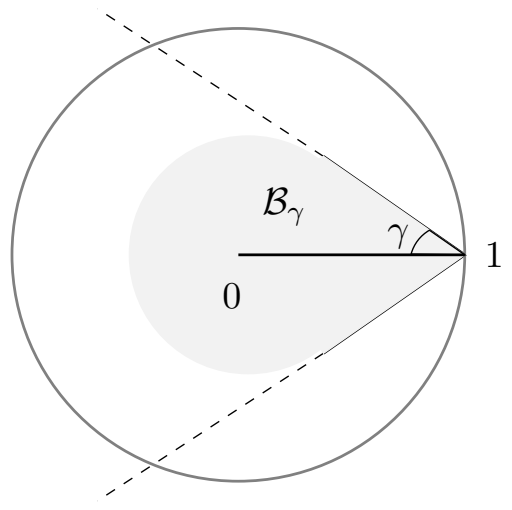

Figure 2.

2.2. Ritt operators: We recommend [19], [21] and [33] references therein for details about Ritt operators.

Definition 2.3 (Ritt operator). For any $\gamma \in\left(0, \frac{\pi}{2}\right)$, let $\mathcal{B}_{\gamma}$ (Stolz domain of angle $\gamma$ ) be the interior of the convex hull of 1 and the disc D(0, $\sin \gamma)$ (FIGURE 2). An operator $T: X \rightarrow X$ is said to be a Ritt operator of type $\alpha \in\left(0, \frac{\pi}{2}\right)$ if

(i) $\sigma(T) \subseteq \overline{\mathcal{B}_{\alpha}}$. (see FIGURE 2)

(ii) For any $\beta \in\left(\alpha, \frac{\pi}{2}\right)$, the set $\left\{(1-\lambda) R(\lambda, T): \lambda \in \mathbb{C} \backslash \overline{\mathcal{B}_{\beta}}\right\}$ is bounded.

Let $\gamma_{i} \in\left(0, \frac{\pi}{2}\right), 1 \leq i \leq n$. Let $H_{0}^{\infty}\left(\prod_{i=1}^{n} \mathcal{B}_{\gamma_{i}}\right)$ be the set of all bounded holomorphic functions $\phi: \prod_{i=1}^{n} \mathcal{B}_{\gamma_{i}} \rightarrow \mathbb{C}$, such that

$$
\left|\phi\left(\lambda_{1}, \ldots, \lambda_{n}\right)\right| \leq c \prod_{i=1}^{n}\left|1-\lambda_{i}\right|^{s}, \text { for }\left(\lambda_{1}, \ldots, \lambda_{n}\right) \in \prod_{i=1}^{n} \mathcal{B}_{\gamma_{i}}
$$

for some constants $c, s>0$, depending only on $\phi$. Let $\Gamma_{\mathcal{B}_{\beta}}$ denote the boundary of $\mathcal{B}_{\beta}$ oriented counterclockwise. Suppose $\mathbf{T}=\left(T_{1}, \ldots, T_{n}\right)$ is a commuting tuple of Ritt operators such that each $T_{i}$ is a Ritt operator of type $\alpha_{i} \in\left(0, \frac{\pi}{2}\right)$ for $1 \leq i \leq n$. Suppose $\beta_{i} \in\left(\alpha_{i}, \gamma_{i}\right), 1 \leq i \leq n$. We define

$$
\phi(\mathbf{T}):=\left(\frac{1}{2 \pi i}\right)^{n} \int_{\prod_{i=1}^{n} \Gamma_{\mathcal{B}_{\beta_{i}}}} \phi\left(\lambda_{1}, \ldots, \lambda_{n}\right) \prod_{i=1}^{n} R\left(\lambda_{i}, A_{i}\right) d \lambda_{i} .
$$

The above integral is absolutely convergent due to adequate decay on the resolvent operators. By Cauchy's theorem it is independent of $\beta_{i}, 1 \leq i \leq n$.

Definition 2.4. We say that $\boldsymbol{T}$ admits a joint bounded $H^{\infty}\left(\prod_{i=1}^{n} \mathcal{B}_{\gamma_{i}}\right)$ functional calculus if

$$
\|\phi(\boldsymbol{T})\|_{X \rightarrow X} \leq C\|\phi\|_{\infty, \prod_{i=1}^{n} \mathcal{B}_{\gamma_{i}}}
$$

for some positive constant $C>0$, which is independent of $\phi$ and $\phi \in H_{0}^{\infty}\left(\prod_{i=1}^{n} \mathcal{B}_{\gamma_{i}}\right)$.

In the spirit of [5] and [4] we investigate necessary and sufficient conditions for commuting tuple of Ritt operators to admit a joint bounded functional calculus. We prove the following useful transfer result.

Theorem 2.5 (Transfer principle). Suppose $\mathbf{T}=\left(T_{1}, \ldots, T_{n}\right)$ is a commuting tuple of Ritt operators on $X$. Let us denote the sectorial operators $A_{i}=I_{X}-T_{i}$ for $1 \leq i \leq n$. Then, the following are equivalent.

1. There exist $\gamma_{i} \in\left(0, \frac{\pi}{2}\right), 1 \leq i \leq n$, such that for all $1 \leq k \leq n$, and $1 \leq i_{1}<\cdots<i_{k} \leq n$, the tuples $\left(T_{i_{1}}, \ldots, T_{i_{k}}\right)$ admit a joint bounded $H^{\infty}\left(\mathcal{B}_{\gamma_{i_{1}}} \times \cdots \times \mathcal{B}_{\gamma_{i_{k}}}\right)$ functional calculus 
2. There exist $\theta_{i} \in\left(0, \frac{\pi}{2}\right), 1 \leq i \leq n$, such that for all $1 \leq k \leq n$, and $1 \leq i_{1}<\cdots<i_{k} \leq n$, the tuples $\left(A_{i_{1}}, \ldots, A_{i_{k}}\right)$ admit a joint bounded $H^{\infty}\left(\Sigma_{\theta_{i_{1}}} \times \cdots \times \Sigma_{\theta_{i_{k}}}\right)$ functional calculus.

The above mentioned result is a mulitivariable generalisation of a similar transfer principle, proved in [21] and can be exploited to transfer known results about sectorial operators to Ritt operators.

The existence of bounded functional calculus is deeply connected to the notion of dilation. For instance, if $T$ is a contraction on a Hilbert space, then the classical result of Sz-Nagy and Foias (see [34], Chapter 1) says that $T$ has a unitary dilation. Ando [3] showed that any commuting couple of contractions on a Hilbert space admits a joint unitary dilation on a common Hilbert space. Nagy-Foias and Ando's dilation theorem can be used to obtain von Neumann inequality in one and two variables respectively. However, there are examples [14], [35] of three commuting contractions on a Hilbert space which fail to have a joint unitary dilation. Moving out of the realm of Hilbert spaces, Akocglu and Sucheston [1] proved that any positive contraction on an $L^{p}$-space has a onto isometric dilation, $1<p \neq 2<\infty$, which in turn shows that this class of contractions satisfy the Matsaev's conjecture. We refer [7], [8] and [27] and references therein for more information in this direction and [32] for multivariate generalisations. In [5] and [4], the authors provided a characterisation of bounded functional calculus for Ritt operators in terms of loose dilation, which we define below.

Definition 2.6. Let $1<p \neq 2<\infty$. Suppose $\mathbf{T}=\left(T_{1}, \ldots, T_{n}\right)$ be a commuting tuple of bounded operators on $L^{p}(\Omega)$. We say that the $n$-tuple $\mathbf{T}$ admits a joint isometric loose dilation, if there exists a measure space $\Omega^{\prime}$, a commuting tuple of onto isometries $\mathbf{U}=\left(U_{1}, \ldots, U_{n}\right)$, on $L^{p}\left(\Omega^{\prime}\right)$, together with two bounded operators $\mathcal{Q}: L^{p}\left(\Omega^{\prime}\right) \rightarrow L^{p}(\Omega)$ and $\mathcal{J}: L^{p}(\Omega) \rightarrow L^{p}\left(\Omega^{\prime}\right)$, such that

$$
T_{1}^{i_{1}} \cdots T_{n}^{i_{n}}=\mathcal{Q} U_{1}^{i_{1}} \cdots U_{n}^{i_{n}} \mathcal{J}
$$

for all $i_{1}, \ldots, i_{n} \in \mathbb{N}_{0}$.

In the case of Hilbert space and for any commuting tuple $\mathbf{T}=\left(T_{1}, \ldots, T_{n}\right)$ of bounded operators on $\mathcal{H}$, we say that the $n$-tuple $\mathbf{T}$ admits a joint isometric loose dilation, if there exists a Hilbert space space $\mathcal{K}$, a commuting tuple of onto isometries $\mathbf{U}=\left(U_{1}, \ldots, U_{n}\right)$, on $\mathcal{K}$, together with two bounded operators $\mathcal{Q}: \mathcal{K} \rightarrow \mathcal{H}$ and $\mathcal{J}: \mathcal{H} \rightarrow \mathcal{K}$, such that

$$
T_{1}^{i_{1}} \cdots T_{n}^{i_{n}}=\mathcal{Q} U_{1}^{i_{1}} \cdots U_{n}^{i_{n}} \mathcal{J}
$$

for all $i_{1}, \ldots, i_{n} \in \mathbb{N}_{0}$.

We prove the following theorem, which can be realised as a multivariate generalisation of Theorem (4.1) of [5].

Theorem 2.7. Let $1<p<\infty$. Let $X$ be a reflexive Banach space such that both $X$ and $X^{*}$ have finite cotype. Let $\mathbf{T}=\left(T_{1}, \ldots, T_{n}\right)$ be a commuting tuple of bounded operators on $X$ such that each $T_{i}$ is Ritt operator and admits a bounded $H^{\infty}$-functional calculus. Then, there exists a measure space $\Omega$, a commuting tuple of isometric isomorphisms $\mathbf{U}=\left(U_{1}, \ldots, U_{n}\right)$ on $L^{p}(\Omega, X)$, together with two bounded operators $\mathcal{Q}: L^{p}(\Omega, X) \rightarrow X$ and $\mathcal{J}: X \rightarrow L^{p}(\Omega, X)$, such that

$$
T_{1}^{i_{1}} \cdots T_{n}^{i_{n}}=\mathcal{Q} U_{1}^{i_{1}} \cdots U_{n}^{i_{n}} \mathcal{J}, \text { for all } i_{1}, \ldots, i_{n} \in \mathbb{N}_{0} .
$$

In addition, we have

1. If $X$ is an ordered Banach space, the maps $U_{j}$ 's can be chosen as positive operators, $1 \leq j \leq n$.

2. If $X$ is a closed subspace of an $L^{p}$-space, then $L^{p}(\Omega, X)$ is again a closed subspace of an $L^{p}$-space and the maps $U_{j}$ 's can be chosen to be restrictions of a commuting tuple of positive isometric isomorphisms on an $L^{p}$-space, $1 \leq j \leq n$. 
3. If $X$ is an $S Q_{p}$ space, then the Banach space $L^{p}(\Omega, X)$ is again an $S Q_{p}$ space and the maps $U_{j}$ 's can be chosen to be compressionss of a commuting tuple of positive isometric isomorphisms on an $L^{p}$-space, $1 \leq j \leq n$.

As far as we know there is no Ando like result for commuting positive operators on $L^{p}$-spaces. However, the following result states that if $\left(T_{1}, T_{2}\right)$ are commuting tuple of positive contractions and one of them is Ritt and admits a bounded $H^{\infty}$-functional calculus, the tuple admits a joint isometric loose dilation. In general for $n$-tuple we have the following result whose proof will be similar to the proof of Theorem (2.7).

Theorem 2.8. Let $1<p<\infty$. Let $\mathbf{T}=\left(T_{1}, \ldots, T_{n}\right)$ be a commuting tuple of bounded operators on $L^{p}(\Omega)$ such that

1. $T_{1}$ admits a loose dilation,

2. Each $T_{i}$ is Ritt operator and $T_{i}$ admits a bounded $H^{\infty}$-functional calculus for $2 \leq i \leq n$.

Then $\mathbf{T}$ admits a joint isometric loose dilation.

The concept of loose dilation leads to the notion of $p$-polynomially boundedness and $p$ completely polynomially boundedness (see [4]). We need the following definitions.

For $1 \leq j \leq n$, we define the $j$-th left shift operator on $\ell_{p}\left(\mathbb{Z}^{n}\right)$ as

$$
\left(S_{j}(a)\right)_{i_{1}, \ldots, i_{j}, \ldots, i_{n}}:=a_{i_{1}, \ldots, i_{j}-1, \ldots, i_{n}}, a \in \ell_{p}\left(\mathbb{Z}^{n}\right) .
$$

Definition 2.9 (Jointly $p$-polynomially bounded). Let $1<p<\infty$. Let $\mathbf{T}=\left(T_{1}, \ldots, T_{n}\right)$ be a commuting tuple of bounded operators on $X$. We say that the $n$-tuple $\mathbf{T}$ is jointly p-polynomially bounded, if there exists a $K>0$ (independent of $P$ ), such that

$$
\|P(\mathbf{T})\|_{X \rightarrow X} \leq K\|P(\mathcal{S})\|_{\ell_{p}\left(\mathbb{Z}^{n}\right) \rightarrow \ell_{p}\left(\mathbb{Z}^{n}\right)}
$$

for any polynomial $P \in \mathbb{C}\left[Z_{1}, \ldots, Z_{n}\right]$, where $\mathcal{S}:=\left(S_{1}, \ldots ., S_{n}\right)$ is the commuting tuple of left shift operators on $\ell_{p}\left(\mathbb{Z}^{n}\right)$.

Definition 2.10 (Jointly $p$-completely polynomially bounded). Let $1<p<\infty$ and $\boldsymbol{T}=$ $\left(T_{1}, \ldots, T_{n}\right)$ be a commuting tuple of bounded operators on $X$. We say that $\boldsymbol{T}$ is jointly $p$ completely polynomially bounded, if for all $N \in \mathbb{N}$, there exists a constant $C>0$, such that

$$
\left\|\left(P_{i, j}(\boldsymbol{T})\right)\right\|_{L^{p}([N], X) \rightarrow L^{p}([N], X)} \leq C\left\|\left(P_{i, j}(\mathcal{S})\right)\right\|_{L^{p}\left([N], \ell^{p}\left(\mathbb{Z}^{n}\right)\right) \rightarrow L^{p}\left([N], \ell^{p}\left(\mathbb{Z}^{n}\right)\right)},
$$

where $P_{i, j} \in \mathbb{C}\left[Z_{1}, \ldots, Z_{n}\right], 1 \leq i, j \leq N$ and $[N]=:\{1, \ldots, N\}$ with counting measure.

Note that, in the case of single variable and operators on Hilbert space, the above notions of 2-polynomially boundedness and 2-completely polynomially boundedness agree with the usual notions of polynomially boundedness and completely polynomially boundedness respectively. We refer [29] for a detailed exposition regarding these concepts.

We require the important notion of $R$-boundedness. Let us consider the probability space $\Omega_{0}=\{ \pm 1\}^{\mathbb{Z}}$. For any integer $k \in \mathbb{Z}$, denote the $k$-th coordinate function as $\epsilon_{k}(\omega)=\omega_{k}$, where $\omega=\left(\omega_{j}\right)_{j \in \mathbb{Z}} \in \Omega_{0}$. The sequence of i.i.d. random variables $\left(\epsilon_{k}\right)_{k \geq 0}$ is called the Rademacher system on the probability space $\Omega_{0}$. For $1 \leq p<\infty$ we denote the $\operatorname{Banach}$ space $\operatorname{Rad}_{p}(X) \subseteq$ $L^{p}\left(\Omega_{0}, X\right)$ to be the closure of the set $\operatorname{span}\left\{\epsilon_{k} \otimes \omega_{k}: k \in \mathbb{Z}, x_{k} \in X\right\}$ in the Bochner space $L^{p}\left(\Omega_{0}, X\right)$. For $p=2$ we simply denote $\operatorname{Rad}(X)$.

Let $E \subseteq B(X)$ be a set of bounded operators in $X$. We say that $E$ is $R$-bounded provided, there exists a constant $C>0$, such that for any finite sequence $\left(T_{k}\right)_{k=0}^{N}$ of $E$ and a finite sequence $\left(x_{k}\right)_{k=0}^{N}$ of $X$

$$
\left\|\sum_{k=0}^{N} \epsilon_{k} \otimes T_{k}\left(x_{k}\right)\right\|_{\operatorname{Rad}(\mathrm{X})} \leq C\left\|\sum_{k=0}^{N} \epsilon_{k} \otimes x_{k}\right\|_{\operatorname{Rad}(\mathrm{X})} .
$$


We also need the notions of $R$-Ritt and $R$-sectorial operators, which one obtains by replacing bounded by $R$-bounded in Definition (2.1) and Definition (2.3) respectively. We suggest [6], [38] and references therein for more about $R$-boundedness and its applications.

Following result provides a characterisation of joint bounded functional calculus for commuting tuple of Ritt operators on $L^{p}$-spaces, for $1<p<\infty$, where each of them admits a bounded functional calculus.

Theorem 2.11. Let $1<p \neq 2<\infty$ and $\mathbf{T}=\left(T_{1}, \ldots, T_{n}\right)$ be a commuting tuple of Ritt operators on $L^{p}(\Omega)$. Then the following assertions are equivalent.

1. There exist $\gamma_{i} \in\left(0, \frac{\pi}{2}\right), 1 \leq i \leq n$, such that for all $1 \leq k \leq n$, and $1 \leq i_{1}<\cdots<i_{k} \leq n$, the tuples $\left(T_{i_{1}}, \ldots, T_{i_{k}}\right)$ admit a joint bounded $H^{\infty}\left(\mathcal{B}_{\gamma_{i_{1}}} \times \cdots \times \mathcal{B}_{\gamma_{i_{k}}}\right)$ functional calculus

2. Each $T_{i}, 1 \leq i \leq n$ is $R$-Ritt and $\mathbf{T}$ admits a joint isometric loose dilation.

3. Each $T_{i}, 1 \leq i \leq n$ is $R$-Ritt and $\mathbf{T}$ is jointly $p$-completely polynomially bounded.

4. Each $T_{i}, 1 \leq i \leq n$ is $R$-Ritt and $\mathbf{T}$ is jointly p-polynomially bounded.

5. Each $T_{i}, 1 \leq i \leq n$ is $R$-Ritt and $I-T_{i}$ admits a bounded $H^{\infty}\left(\Sigma_{\theta_{i}}\right)$ functional calculus for $\theta_{i} \in(0, \pi)$, for each $1 \leq i \leq n$.

For single Ritt operators we refer [4].

2.3. Similarity problem: If an operator $T$ on a Hilbert space is similar to a contraction then it is polynomially bounded. The converse was a long-standing open problem for many years (see [15]). Finally, Pisier [30] in 1997 constructed an example to show that the converse does not hold. Essentially, he produced an operator which is polynomially bounded but not completely polynomially bounded (In between Paulsen [26] had shown that $T$ is similar to contraction if and only if it is completely polynomially bounded). For multivariable case, it is natural to ask if for any commuting tuple of bounded operators $\mathbf{T}=\left(T_{1}, \ldots, T_{n}\right), T_{i} \in B(\mathcal{H})$, $1 \leq i \leq n$, such that each $T_{i}$ is similar to a contraction will automatically imply that $T_{i}$ 's are jointly similar to a commuting tuple of contractions, i.e., if there exists an invertible map $S$ such that $\left(S T_{1} S^{-1}, \ldots, S T_{n} S^{-1}\right)$ is a commuting tuple of contractions ? Again, Pisier [31] (also see [28]) answered it negatively by showing existence of two commuting contractions $\left(T_{1}, T_{2}\right)$ on a Hilbert space, $T_{i}$ being similar to a contraction, $1 \leq i \leq 2$, but $T_{1} T_{2}$ is not even polynomially bounded. In this context, one can also ask for examples of class of operators, for which the joint similarity problem has a positive solution. It is known that the Halmos's similarity problem has a positive answer in the class of Ritt operators [10], [21], [22]. Following result asserts an affirmative answer for joint similarity problem in the class of Ritt operators.

Theorem 2.12. Let $\mathbf{T}=\left(T_{1}, \ldots, T_{n}\right)$ be a commuting tuple of Ritt operators on a Hilbert space $\mathcal{H}$. Then, the following assertions are equivalent.

1. Each $T_{i}$ is similar to a contraction, $1 \leq i \leq n$.

2. For all $1 \leq k \leq n$, and $1 \leq i_{1}<\cdots<i_{k} \leq n$, the tuples $\left(T_{i_{1}}, \ldots, T_{i_{k}}\right)$ admit a joint bounded $H^{\infty}$-functional calculus.

3. The tuple $\mathbf{T}=\left(T_{1}, \ldots, T_{n}\right)$ is jointly similar to a commuting n-tuple of contractions.

The above theorem can be interpreted as a Hilbert space variant of Theorem (2.11). Also, it is natural to ask for examples of couple of commuting operators $\left(T_{1}, T_{2}\right)$ on general Banach spaces, such that each of which is $p$-polynomially bounded (resp. completely $p$-polynomially bounded) but not jointly $p$-polynomially bounded (resp. jointly $p$-completely polynomially bounded). We do not have such example. However, in view of Theorem (2.11) we have a positive answer for Ritt operators on $L^{p}$-spaces which are positive contractions, $1<p \neq 2<\infty$.

\section{Proof of the results:}

Proof of Theorem (2.5): 


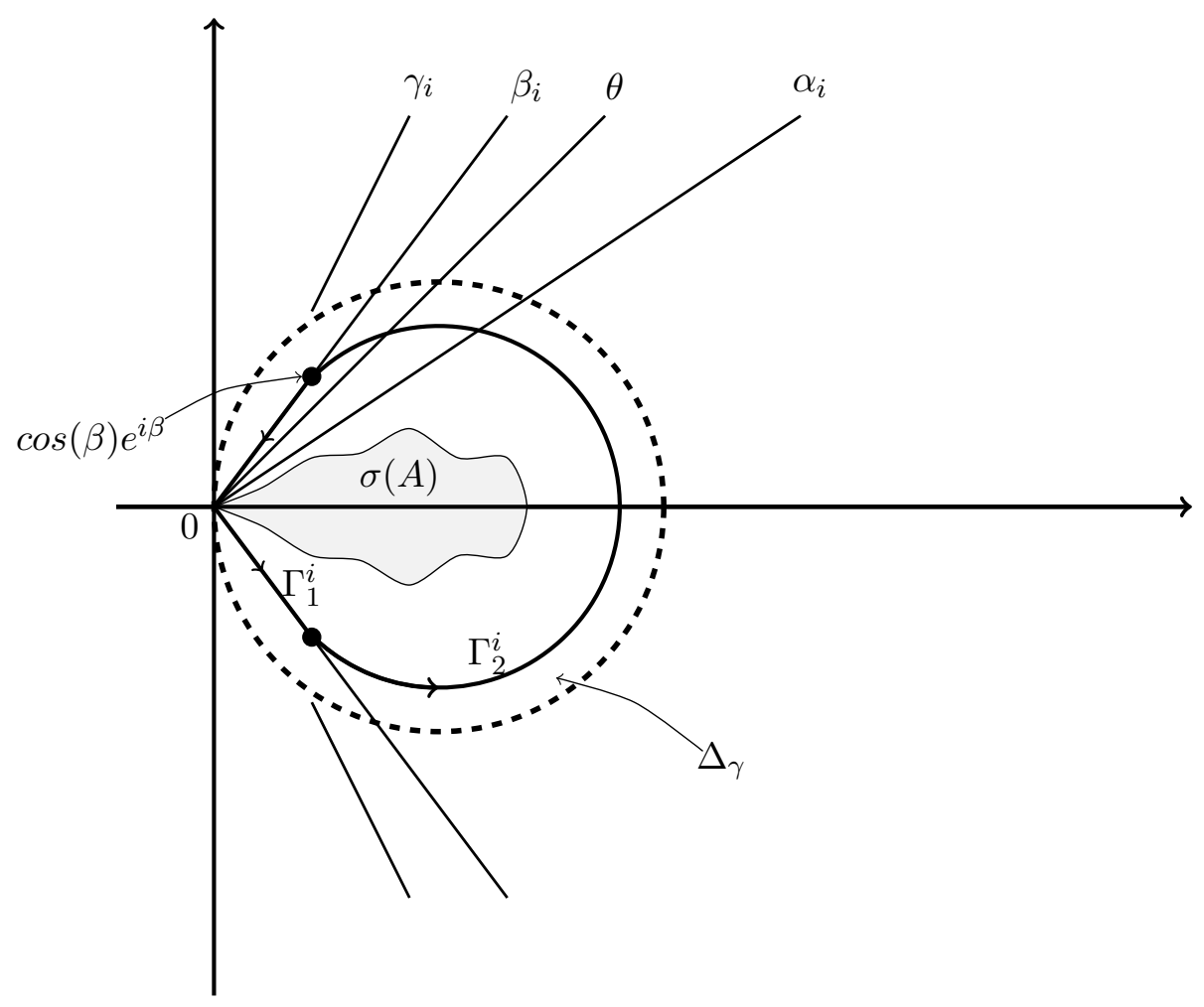

FiguRE 3.

Let us denote $\Delta_{\gamma}:=1-\mathcal{B}_{\gamma}$ for $\gamma \in\left(0, \frac{\pi}{2}\right)$. Fix $\gamma_{i} \in\left(0, \frac{\pi}{2}\right)$ and $\beta_{i} \in\left(0, \gamma_{i}\right)$, for $1 \leq i \leq n$. Let $\alpha_{i}<\theta<\beta_{i}$. Let $\Gamma_{1}^{i}$ be the contour connecting $\cos \beta_{i} \exp \left(i \beta_{i}\right)$ to 0 and 0 to $\cos \beta_{i} \exp \left(-i \beta_{i}\right)$ via straight lines, and $\Gamma_{2}^{i}$ be the contour going from $\cos \beta \exp \left(-i \beta_{i}\right)$ to $\cos \beta \exp \left(i \beta_{i}\right)$ along the circle with centre at 1 and radius $\sin \beta$ in the counterclockwise direction (see FIGURE 3 ). Define the contour $\Gamma^{i}:=\Gamma_{1}^{i}+\Gamma_{2}^{i}$. Let $f \in H^{\infty}\left(\prod_{i=1}^{n} \Delta_{\gamma_{i}}\right)$ satisfying $\left|f\left(z_{1}, \ldots, z_{n}\right)\right| \leq c_{1} \prod_{i=1}^{n}\left|z_{i}\right|^{s}$ for some $s>0$. For $j_{k} \in\{1,2\}, 1 \leq k \leq n$, let us define (on an appropriate domain)

$$
f_{j_{1}, \ldots, j_{n}}\left(z_{1}, \ldots, z_{n}\right):=\left(\frac{1}{2 \pi i}\right)^{n} \int_{\Gamma_{j_{1}}^{1}} \cdots \int_{\Gamma_{j_{n}}^{n}} \frac{f\left(\lambda_{1}, \ldots, \lambda_{n}\right)}{\prod_{i=1}^{n}\left(\lambda_{i}-z_{i}\right)} d \lambda_{1} \cdots d \lambda_{n}
$$

Observe that, for $\left(z_{1}, \ldots, z_{n}\right) \in \prod_{i=1}^{n} \mathcal{B}_{\beta_{i}}$ by Cauchy's theorem,

$$
f\left(z_{1}, \ldots, z_{n}\right)=\sum_{1 \leq j_{1}, \ldots, j_{n} \leq 2} f_{j_{1}, \ldots, j_{n}}\left(z_{1}, \ldots, z_{n}\right)
$$

Let us fix a tuple $\left(j_{1}, \ldots, j_{n}\right)$, where not all $j_{k}$ 's take the same value. Define the following affine maps on $\mathbb{C}^{n}$ as

$$
\mathcal{P}_{i} \mathfrak{F}\left(j_{1}, \ldots, j_{n}\right)\left(z_{1}, \ldots, z_{n}\right):= \begin{cases}z_{i}, & j_{i}=1 \\ 0, & j_{i} \neq 1\end{cases}
$$


where $\mathcal{P}_{i}: \mathbb{C}^{n} \rightarrow \mathbb{C}^{n}$ is the $i$-th projection map, for $1 \leq i \leq n$. Define

$$
\begin{aligned}
F_{j_{1}, \ldots, j_{n}}\left(z_{1}, \ldots, z_{n}\right) & :=\frac{\left(f_{j_{1}, \ldots, j_{n}}\left(\mathfrak{F}\left(j_{1}, \ldots, j_{n}\right)\right)\left(z_{1}, \ldots, z_{n}\right)\right.}{\prod_{\left\{i, j_{i}=2\right\}}\left(1+z_{i}\right)}, \\
F_{1, \ldots, 1}\left(z_{1}, \cdots, z_{n}\right) & :=f_{1, \ldots, 1}\left(z_{1}, \cdots, z_{n}\right) \\
F_{2, \ldots, 2}\left(z_{1}, \cdots, z_{n}\right) & :=\frac{f_{2, \ldots, 2}(0, \ldots, 0)}{\prod_{i=1}^{n}\left(1+z_{i}\right)} .
\end{aligned}
$$

First, we will prove two lemmas, which are essential for the proof of Theorem (2.5). We need the following single variable version of Lemma (3.2) which was part of Proposition (4.1) in [21].

Lemma 3.1 ([21]). Let $0<\theta<\beta_{1}<\gamma_{1}<\frac{\pi}{2}$. Let $f \in H^{\infty}\left(\Delta_{\gamma_{1}}\right)$ satisfying $\left|f\left(z_{1}\right)\right| \leq c_{1}\left|z_{1}\right|^{s}$ for some $c, s>0$. Let us define

$$
g\left(z_{1}\right)=F_{1}\left(z_{1}\right)+\frac{1}{1+z_{1}} F_{2}(0)
$$

where

$$
F_{j}\left(z_{1}\right)=\frac{1}{2 \pi i} \int_{\Gamma_{j}^{1}} \frac{f\left(\lambda_{1}\right)}{\lambda_{1}-z_{1}} d \lambda_{1}, \text { for } j=1,2 .
$$

Then $g \in H_{0}^{\infty}\left(\Sigma_{\theta}\right)$ and $\|g\|_{\infty, \Sigma_{\theta}} \leq C\|f\|_{\infty, \Delta_{\gamma_{1}}}$.

Throughout this section we will follow notations as in the beginning of this section.

Lemma 3.2. Let us define the following auxiliary function

$$
g\left(z_{1}, \ldots, z_{n}\right)=\sum_{1 \leq j_{1}, \ldots, j_{n} \leq 2} F_{j_{1}, \ldots, j_{n}}\left(z_{1}, \ldots, z_{n}\right) .
$$

Let $\theta \in\left(0, \beta_{i}\right), 1 \leq i \leq n$. Then $g \in H_{0}^{\infty}\left(\Sigma_{\theta}^{n}\right)$.

Proof. We will give the proof for $n=2$ to avoid notational complexity. For general $n$ the proof is in the same spirit.

It is clear that $g$ is holomorphic on appropriate domain. To establish the lemma, we need to consider the following cases.

Case 1: Both $\left|z_{1}\right|$ and $\left|z_{2}\right|$ are large. We use triangle inequality in (6) to obtain the following estimate on $g$.

$$
\begin{aligned}
\left|g\left(z_{1}, z_{2}\right)\right| \leq & \frac{1}{4 \pi^{2}\left|z_{1}\right|\left|z_{2}\right|}\left|\int_{\Gamma_{1}^{1}} \int_{\Gamma_{1}^{2}} \frac{f\left(\lambda_{1}, \lambda_{2}\right)}{\left(\lambda_{1} / z_{1}-1\right)\left(\lambda_{2} / z_{2}-1\right)} d \lambda_{1} d \lambda_{2}\right| \\
& +\frac{1}{4 \pi^{2}\left|z_{1}\right|\left|1+z_{2}\right|}\left|\int_{\Gamma_{1}^{1}} \int_{\Gamma_{2}^{2}} \frac{f\left(\lambda_{1}, \lambda_{2}\right)}{\left(\lambda_{1} / z_{1}-1\right) \lambda_{2}} d \lambda_{1} d \lambda_{2}\right| \\
& +\frac{1}{4 \pi^{2}\left|z_{2}\right|\left|1+z_{1}\right|}\left|\int_{\Gamma_{2}^{1}} \int_{\Gamma_{1}^{2}} \frac{f\left(\lambda_{1}, \lambda_{2}\right)}{\lambda_{1}\left(\lambda_{2} / z_{2}-1\right)} d \lambda_{1} d \lambda_{2}\right| \\
& +\left|\frac{1}{\left(1+z_{1}\right)\left(1+z_{2}\right)} f_{4}(0,0)\right| .
\end{aligned}
$$

Therefore, in this case $\left|g\left(z_{1}, z_{2}\right)\right| \leq c_{2}\left|z_{1}\right|^{-1}\left|z_{2}\right|^{-1}$ for some constant $c_{2}>0$ which is independent of $z_{1}, z_{2}$.

Case 2: $\left|z_{2}\right|$ is large and $\left|z_{1}\right|$ small. We group $F_{1,1}$ and $F_{2,1}$ in (6) to obtain the following expression

$$
-\frac{1}{4 \pi^{2}}\left(\int_{\Gamma_{1}^{2}} \frac{1}{\lambda_{2}-z_{2}}\left(\int_{\Gamma_{1}^{1}} \frac{f\left(\lambda_{1}, \lambda_{2}\right)}{\left(\lambda_{1}-z_{1}\right)} d \lambda_{1}+\frac{1}{1+z_{1}} \int_{\Gamma_{2}^{1}} \frac{f\left(\lambda_{1}, \lambda_{2}\right)}{\lambda_{1}} d \lambda_{1}\right) d \lambda_{2}\right) .
$$


For a fixed $\lambda_{2}$ let us define, $\tilde{f}^{\lambda_{2}}(z)=f\left(z_{1}, \lambda_{2}\right)$. Applying Lemma (3.1) to the function $\tilde{f}^{\lambda_{2}}$, the term inside the bracket of (7) will produce a factor of $\left|z_{1}\right|^{s}$ for some $s>0$ and as $\left|z_{2}\right|$ is large we will get a factor of $\frac{1}{\mid z_{2}}$. Hence, we have the estimate $\left|F_{1,1}\left(z_{1}, z_{2}\right)+F_{2,1}\left(0, z_{2}\right)\right| \leq c_{3}\left|z_{1}\right|^{s}\left|z_{2}\right|^{-1}$. Similarly, we group $F_{1,2}$ and $F_{2,2}$ in (6) to obtain appropriate bound for $g$.

Case 3: $\left|z_{1}\right|$ is large and $\left|z_{2}\right|$ small. This is similar to the previous case.

Case 4: Both $\left|z_{1}\right|$ and $\left|z_{2}\right|$ are small. We apply Cauchy's theorem to obtain the following identity

$$
\begin{aligned}
g\left(z_{1}, z_{2}\right)= & f\left(z_{1}, z_{2}\right)-\left(f_{1,2}\left(z_{1}, z_{2}\right)-\frac{1}{1+z_{2}} f_{1,2}\left(z_{1}, 0\right)\right) \\
& -\left(f_{2,1}\left(z_{1}, z_{2}\right)-\frac{1}{1+z_{1}} f_{2,1}\left(0, z_{2}\right)\right) \\
& -\left(f_{2,2}\left(z_{1}, z_{2}\right)-\frac{1}{\left(1+z_{1}\right)\left(1+z_{2}\right)} f_{2,2}(0,0)\right) \\
= & I_{1}-I_{2}-I_{3}-I_{4} .
\end{aligned}
$$

Let us rewrite and notice that

$$
\begin{aligned}
I_{2}= & \left(f_{1,2}\left(z_{1}, z_{2}\right)-f_{1,2}\left(z_{1}, 0\right)\right)+\frac{z_{2}}{1+z_{2}} f_{1,2}\left(z_{1}, 0\right) \\
= & \frac{1}{2 \pi i} \int_{\Gamma_{2}^{2}} \frac{z_{2} f\left(z_{1}, \lambda_{2}\right)}{\lambda_{2}\left(\lambda_{2}-z_{2}\right)} d \lambda_{2}+\frac{1}{4 \pi^{2}} \int_{\Gamma_{2}^{1}} \int_{\Gamma_{2}^{2}} \frac{z_{2} f\left(\lambda_{1}, \lambda_{2}\right)}{\lambda_{2}\left(\lambda_{1}-z_{1}\right)\left(\lambda_{2}-z_{2}\right)} d \lambda_{1} d \lambda_{2} \\
& \left.+\frac{z_{2}}{1+z_{2}} f_{1,2} z_{1}, 0\right) .
\end{aligned}
$$

By an analogous computation, we derive

$$
\begin{aligned}
I_{3}= & \frac{1}{2 \pi i} \int_{\Gamma_{2}^{1}} \frac{z_{1} f\left(\lambda_{1}, z_{2}\right)}{\lambda_{1}\left(\lambda_{1}-z_{2}\right)} d \lambda_{1} \\
& +\frac{1}{4 \pi^{2}} \int_{\Gamma_{2}^{1}} \int_{\Gamma_{2}^{2}} \frac{z_{1} f\left(\lambda_{1}, \lambda_{2}\right)}{\lambda_{1}\left(\lambda_{1}-z_{1}\right)\left(\lambda_{2}-z_{2}\right)} d \lambda_{1} d \lambda_{2}+\frac{z_{1}}{1+z_{1}} f_{2,1}\left(z_{1}, 0\right) .
\end{aligned}
$$

Now we observe the following

$$
\begin{aligned}
& f_{2,2}\left(z_{1}, z_{2}\right)-f_{2,2}(0,0) \\
= & -\frac{1}{4 \pi^{2}}\left(\int_{\Gamma_{2}^{1}} \int_{\Gamma_{2}^{2}} \frac{z_{2} f\left(\lambda_{1}, \lambda_{2}\right)}{\lambda_{2}\left(\lambda_{1}-z_{1}\right)\left(\lambda_{2}-z_{2}\right)} d \lambda_{1} d \lambda_{2}\right. \\
& +\int_{\Gamma_{2}^{1}} \int_{\Gamma_{2}^{2}} \frac{z_{1} f\left(\lambda_{1}, \lambda_{2}\right)}{\lambda_{1}\left(\lambda_{1}-z_{1}\right)\left(\lambda_{2}-z_{2}\right)} d \lambda_{1} d \lambda_{2} \\
& \left.-z_{1} z_{2} \int_{\Gamma_{2}^{1}} \int_{\Gamma_{2}^{2}} \frac{f\left(\lambda_{1}, \lambda_{2}\right)}{\lambda_{2} \lambda_{1}\left(\lambda_{2}-z_{2}\right)\left(\lambda_{1}-z_{1}\right)} d \lambda_{1} d \lambda_{2}\right) .
\end{aligned}
$$


Hence, by adding all the terms we get

$$
\begin{aligned}
g\left(z_{1}, z_{2}\right)= & f\left(z_{1}, z_{2}\right)-\frac{1}{2 \pi i}\left(\int_{\Gamma_{2}^{2}} \frac{z_{2} f\left(z_{1}, \lambda_{2}\right)}{\lambda_{2}\left(\lambda_{2}-z_{2}\right)} d \lambda_{2}+\int_{\Gamma_{2}^{1}} \frac{z_{1} f\left(\lambda_{1}, z_{2}\right)}{\lambda_{1}\left(\lambda_{1}-z_{1}\right)} d \lambda_{1}\right) \\
& -\frac{z_{2}}{1+z_{2}}\left(f_{1,2}\left(z_{1}, 0\right)+\frac{1}{\left(1+z_{1}\right)} f_{2,2}(0,0)\right) \\
& -\frac{z_{1}}{1+z_{1}}\left(f_{2,1}\left(0, z_{2}\right)+\frac{1}{\left(1+z_{2}\right)} f_{2,2}(0,0)\right) \\
& -z_{1} z_{2}\left(\frac{1}{4 \pi^{2}} \int_{\Gamma_{2}^{1}} \int_{\Gamma_{2}^{2}} \frac{f\left(\lambda_{1}, \lambda_{2}\right)}{\lambda_{2} \lambda_{1}\left(\lambda_{2}-z_{2}\right)\left(\lambda_{1}-z_{1}\right)} d \lambda_{1} d \lambda_{2}\right. \\
& \left.+\frac{1}{\left(1+z_{1}\right)\left(1+z_{2}\right)} f_{2,2}(0,0)\right) .
\end{aligned}
$$

Since $\left|z_{1}\right|,\left|z_{2}\right|$ both small, we have for some $s_{1}, s_{2}>0$,

$$
\left|\int_{\Gamma_{2}^{2}} \frac{z_{2} f\left(z_{1}, \lambda_{2}\right)}{\lambda_{2}\left(\lambda_{2}-z_{2}\right)} d \lambda_{2}+\int_{\Gamma_{2}^{1}} \frac{z_{1} f\left(\lambda_{1}, z_{2}\right)}{\lambda_{1}\left(\lambda_{1}-z_{1}\right)} d \lambda_{1}\right| \leq K_{1}\left|z_{1}\right|^{s_{1}}\left|z_{2}\right|^{s_{2}}
$$

where $K_{1}>0$ is a constant which depends on $f$. We apply Lemma (3.1) as we did to handle Case 2 to get a constant $K_{2}>0$ such that

$$
\left|f_{1,2}\left(z_{1}, 0\right)+\frac{1}{\left(1+z_{1}\right)} f_{2,2}(0,0)\right| \leq K_{2}\left|z_{1}\right|^{s} .
$$

Similarly, for some $K_{3}>0$, we will obtain the bound

$$
\left|f_{2,1}\left(0, z_{2}\right)+\frac{1}{\left(1+z_{2}\right)} f_{2,2}(0,0)\right| \leq K_{3}\left|z_{2}\right|^{s} .
$$

Therefore, in view of (8), (9) and (10), we obtain that $g$ has the appropriate bound.

Proof of Theorem (2.5): We only prove (2) implies (1). We also restrict ourselves for $n=2$, for general $n>2$ the proof can be done using induction. Let us assume that the tuple $\mathbf{A}=$ $\left(A_{1}, A_{2}\right)$ admits a joint bounded $H^{\infty}\left(\prod_{i=1}^{2} \Sigma_{\theta_{i}}\right)$ functional calculus and $A_{i}$ admits a bounded $H^{\infty}\left(\Sigma_{\theta_{i}}\right)$ functional calculus for some $\theta_{i} \in\left(0, \frac{\pi}{2}\right), 1 \leq i \leq 2$. Suppose $\sigma\left(A_{i}\right) \subseteq \overline{\Delta_{\alpha_{i}}}, 1 \leq i \leq 2$. We can assume that all $\theta_{i}$ 's are equal and $\theta>\alpha_{i}$ for $1 \leq i \leq 2$. Now we choose $\beta_{i}$ and $\gamma_{i}$ in $\left(0, \frac{\pi}{2}\right)$ so that $\theta<\beta_{i}<\gamma_{i}$ for $1 \leq i \leq 2$.

Let $\phi \in H_{0}^{\infty}\left(\mathcal{B}_{\gamma_{1}} \times \mathcal{B}_{\gamma_{1}}\right)$. Define $f: \Delta_{\gamma_{1}} \times \Delta_{\gamma_{2}} \rightarrow \mathbb{C}$ to be

$$
f\left(z_{1}, z_{2}\right):=\phi\left(1-z_{1}, 1-z_{2}\right) .
$$

Now we define an auxilliary function $g$ as in (6). Since $g \in H_{0}^{\infty}\left(\Sigma_{\theta} \times \Sigma_{\theta}\right)$ by Lemma (3.2) we can define $g\left(A_{1}, A_{2}\right)$ by (1). Let us define

$$
\begin{gathered}
f_{1,1}\left(A_{1}, A_{2}\right):=g\left(A_{1}, A_{2}\right)-\left(I+A_{2}\right)^{-1} f_{1,2}\left(A_{1}, 0\right)-\left(I+A_{1}\right)^{-1} f_{2,1}\left(0, A_{2}\right) \\
-\left(I+A_{1}\right)^{-1}\left(I+A_{2}\right)^{-1} f_{2,2}(0,0)
\end{gathered}
$$

where in $(11) f_{1,2}\left(A_{1}, 0\right)$ and $f_{2,1}\left(0, A_{2}\right)$ are defined as the following. Consider

$$
\widetilde{g}\left(z_{1}\right)=f_{1,2}\left(z_{1}, 0\right)+\frac{1}{1+z_{1}} f_{2,2}(0,0) .
$$

By Lemma (3.1) $\widetilde{g} \in H_{0}^{\infty}\left(\Sigma_{\theta}\right)$. Therefore, $\widetilde{g}\left(A_{1}\right)$ can be defined as in (1). Thus, we can define

$$
f_{1,2}\left(A_{1}, 0\right):=\widetilde{g}\left(A_{1}\right)-\left(I+A_{1}\right)^{-1} f_{2,2}(0,0) .
$$


We define $f_{2,1}\left(0, A_{2}\right)$ in a similar manner. Since $A_{1}$ admits a bounded functional calculus, we have the estimate $\left\|\widetilde{g}\left(A_{1}\right)\right\| \leq K^{\prime}\|\widetilde{g}\|_{\infty, \Sigma_{\theta}}$. Therefore, it follows immediately from Lemma (3.1) that

$$
\left\|f_{1,2}\left(A_{1}, 0\right)\right\| \leq K^{\prime \prime}\|\widetilde{g}\|_{\infty, \Sigma_{\theta}} \leq K_{2}\|f\|_{\infty, \Delta_{\gamma_{1}} \times \Delta_{\gamma_{2}}} .
$$

Similarly, we get the appropriate bound for $f_{2,1}$,

$$
\left\|f_{2,1}\left(0, A_{2}\right)\right\| \leq K_{3}\|f\|_{\infty, \Delta_{\gamma_{1}} \times \Delta_{\gamma_{2}}} .
$$

Note that the constants $K_{2}$ and $K_{3}$ are independent of $f$. From (11), we get

$$
\left\|f_{1,1}\left(A_{1}, A_{2}\right)\right\| \leq K_{4}\|f\|_{\infty, \Delta_{\gamma_{1}} \times \Delta_{\gamma_{2}}},
$$

for some positive constant $K_{4}$ (independent of $f$ ). For a fixed $\lambda_{2}$ define $\tilde{f}^{\lambda_{2}}\left(z_{1}\right)=f\left(z_{1}, \lambda_{2}\right)$. Note that the function

$$
g^{\lambda_{2}}\left(z_{1}\right):=\frac{1}{2 \pi i}\left(\int_{\Gamma_{1}^{1}} \frac{\tilde{f}^{\lambda_{2}}\left(\lambda_{1}\right)}{\lambda_{1}-z_{1}} d \lambda_{1}+\frac{1}{1+z_{1}} \int_{\Gamma_{2}^{1}} \frac{\tilde{f}^{\lambda_{2}}\left(\lambda_{1}\right)}{\lambda_{1}} d \lambda_{1}\right)
$$

is in $H_{0}^{\infty}\left(\Sigma_{\theta}\right)$. Thus, we use Lemma (3.1) to have

$$
\left\|g^{\lambda_{2}}\left(A_{1}\right)\right\| \leq K_{5}\|f\|_{\infty, \Delta_{\gamma_{1}} \times \Delta_{\gamma_{2}}}
$$

where, $K_{5}>0$ is independent of $f$. Let us define

$$
\begin{aligned}
f_{1,2}\left(A_{1}, A_{2}\right):= & \frac{1}{2 \pi i} \int_{\Gamma_{2}^{2}} g^{\lambda_{2}}\left(A_{1}\right)\left(\lambda_{2}-A_{2}\right)^{-1} d \lambda_{2} \\
& -\left(\frac{1}{2 \pi i}\right)^{2} \int_{\Gamma_{2}^{1}} \int_{\Gamma_{2}^{2}} f\left(\lambda_{1}, \lambda_{2}\right) \lambda_{1}^{-1}\left(\lambda_{2}-A_{2}\right)^{-1} d \lambda_{1} d \lambda_{2} .
\end{aligned}
$$

In above the second integral is defined as Dunford-Riesz functional calculus. Since $\sigma\left(A_{i}\right) \cap \Gamma_{2}^{i}=\emptyset$, $i=1,2$, one can observe that $\inf _{\lambda_{i} \in \Gamma_{2}^{i}}\left\|\lambda_{i} I-A_{i}\right\|>0$ for $1 \leq i \leq 2$. Therefore, by (13) we have an estimate

$$
\left\|f_{1,2}\left(A_{1}, A_{2}\right)\right\| \leq K_{6}\|f\|_{\infty, \Delta_{\gamma_{1}} \times \Delta_{\gamma_{2}}} .
$$

By a similar way one can define $f_{2,1}\left(A_{1}, A_{2}\right)$ and obtain the estimate

$$
\left\|f_{2,1}\left(A_{1}, A_{2}\right)\right\| \leq K_{7}\|f\|_{\infty, \Delta_{\gamma_{1}} \times \Delta_{\gamma_{2}}} .
$$

It is easy to see

$$
\left\|f_{2,2}\left(A_{1}, A_{2}\right)\right\| \leq K_{8}\|f\|_{\infty, \Delta_{\gamma_{1}} \times \Delta_{\gamma_{2}}}
$$

Note that the constants $K_{6}, K_{7}, K_{8}>0$ are independent of $f$. Hence by (11), (14), (15) and (16) we have

$$
\begin{aligned}
\left\|\phi\left(T_{1}, T_{2}\right)\right\| & =\left\|f_{1,1}\left(A_{1}, A_{2}\right)+f_{1,2}\left(A_{1}, A_{2}\right)+f_{2,1}\left(A_{1}, A_{2}\right)+f_{2,2}\left(A_{1}, A_{2}\right)\right\| \\
& \leq K\|\phi\|_{\infty, \mathcal{B}_{\gamma_{1}} \times \mathcal{B}_{\gamma_{2}}},
\end{aligned}
$$

where $K>0$ is independent of $f$. The proof is completed by (17) and [21].

\section{Proof of Theorem (2.7):}

Let $T$ be Ritt operator on $X$. Since $I-T$ is a sectorial operator, one can define via functional calculus the fractional power $(I-T)^{\alpha}$ for any $\alpha>0$. One defines the associated Littlewood-Paley square function

$$
\|x\|_{T, \alpha}:=\left\|\sum_{k=0}^{\infty}(k+1)^{\alpha-\frac{1}{2}} \epsilon_{k} \otimes T^{k}(I-T)^{\alpha} x\right\|_{\operatorname{Rad}(X)},
$$

where $x \in X$. Note that it can happen that $\|x\|_{T, \alpha}=\infty$ for some $x \in X$. 
Now we will give the proof of Theorem (2.7) following the idea of Prof. Christian Le Merdy in a personal communication.

Proof of Theorem (2.7): We proceed by induction on the number of commuting operators. Let us define an operator $\mathrm{u}: L^{p}\left(\Omega_{0}\right) \rightarrow L^{p}\left(\Omega_{0}\right)$ as

$$
\mathrm{u}(f)\left(\left\{\omega_{k}\right\}_{k}\right)=f\left(\left\{\omega_{k-1}\right\}_{k}\right)
$$

for $f \in L^{p}\left(\Omega_{0}\right)$. Clearly, $\mathrm{u}$ is a positive isometric isomorphism. Therefore one can extend $\mathrm{u}$ to an operator $\mathrm{U}:=\mathrm{u} \otimes I_{X}$ on the Banach space $L^{p}\left(\Omega_{0}, X\right)$ as an isometric isomorphism. Since $\mathrm{u}\left(\epsilon_{k}\right)=\epsilon_{k-1}$, for any $\sum_{k} \epsilon_{k} \otimes x_{k} \in \operatorname{Rad}_{p}(X)$, we have $\mathrm{U}\left(\sum_{k} \epsilon_{k} \otimes x_{k}\right)=\sum_{k} \epsilon_{k} \otimes x_{k+1}$. Let $U$ be the isometric isomorphism $I_{X} \oplus \mathrm{U}$ on $X \oplus_{p} L^{p}\left(\Omega_{0}, X\right)$. Note that one can identify $X \oplus_{p} L^{p}\left(\Omega_{0}, X\right)$ as $L^{p}\left(\Omega^{\prime}, X\right)$ for some measure space $\Omega^{\prime}$.

Since the Banach spaces $X$ and $X^{*}$ are of finite cotype and $T_{1}$ admits a bounded $H^{\infty}$ functional calculus, we have the square function estimates [5] $\|x\|_{T_{1}, \frac{1}{2}} \leq C_{1}\|x\|_{X}$ and $\|y\|_{T_{1}^{*}, \frac{1}{2}} \leq C_{2}\|y\|_{X^{*}}$ for all $x \in X, y \in X^{*}$. Again as $T_{1}$ is power bounded and $X$ is reflexive, one can use mean ergodic theorem [18] to have the following decompositions,

$$
\left.X=\operatorname{Ker}\left(I_{X}-T_{1}\right) \oplus \overline{\operatorname{Ran}\left(I_{X}-T_{1}\right.}\right)
$$

and

$$
\left.X^{*}=\operatorname{Ker}\left(I_{X^{*}}-T_{1}^{*}\right) \oplus \overline{\operatorname{Ran}\left(I_{X^{*}}-T_{1}^{*}\right.}\right) .
$$

Also one can notice that for any $\left.x_{0} \in \operatorname{Ker}\left(I_{X}-T_{1}\right), x_{1} \in \overline{\operatorname{Ran}\left(I_{X}-T_{1}\right.}\right)$ and $y_{0} \in \operatorname{Ker}\left(I_{X^{*}}-\right.$ $\left.T_{1}^{*}\right), y_{1} \in \overline{\operatorname{Ran}\left(I_{X^{*}}-T_{1}^{*}\right)}$, we have $\left\langle x_{0}, y_{1}\right\rangle=\left\langle x_{1}, y_{0}\right\rangle=0$. Therefore, from the square function estimates, we can define the linear map

$$
\left.J: \operatorname{Ker}\left(I_{X}-T_{1}\right) \oplus \overline{\operatorname{Ran}\left(I_{X}-T_{1}\right.}\right) \rightarrow X \oplus_{p} L^{p}\left(\Omega_{0}, X\right),
$$

as $J\left(x_{0} \oplus x_{1}\right)=x_{0} \oplus \sum_{k=0}^{\infty} \epsilon_{k} \otimes T_{1}^{k}\left(I_{X}-T_{1}\right)^{\frac{1}{2}} x_{1}$.

Similarly, define

$$
\tilde{J}: \operatorname{Ker}\left(I_{X^{*}}-T_{1}^{*}\right) \oplus \overline{\operatorname{Ran}\left(I_{X^{*}}-T_{1}^{*}\right)} \rightarrow X^{*} \oplus_{p^{\prime}} L^{p^{\prime}}\left(\Omega_{0}, X^{*}\right),
$$

as $\tilde{J}_{1}\left(y_{0} \oplus y_{1}\right)=y_{0} \oplus \sum_{k=0}^{\infty} \epsilon_{k} \otimes T_{1}^{* k}\left(I_{X^{*}}-T_{1}^{*}\right)^{\frac{1}{2}} y_{1}$.

Following the proof of [5] Theorem (4.1), we have that

$$
\left\langle U^{n} J\left(x_{0} \oplus x_{1}\right), \tilde{J}\left(y_{0} \oplus y_{1}\right)\right\rangle=\left\langle x_{0}, x_{1}\right\rangle+\left\langle\left(I_{X}+T_{1}\right)^{-1} T_{1}^{n} x_{1}, y_{1}\right\rangle .
$$

Let us define the operator $\left.\Theta: \operatorname{Ker}\left(I_{X}-T_{1}\right) \oplus \overline{\operatorname{Ran}\left(I_{X}-T_{1}\right.}\right) \rightarrow X \oplus_{p} L^{p}\left(\Omega_{0}, X\right)$ as

$$
\Theta\left(x_{0} \oplus x_{1}\right):=x_{0} \oplus\left(I_{X}+T_{1}\right) x_{1} .
$$

Thereafter, it is easy to check that $\left\langle U^{n} J \Theta\left(x_{0} \oplus x_{1}\right), \tilde{J}\left(y_{0} \oplus y_{1}\right)\right\rangle=\left\langle x_{0}, y_{0}\right\rangle+\left\langle T_{1}^{n} x_{1}, y_{1}\right\rangle$. Define $Q_{1}=\tilde{J}^{*}$ and $J_{1}=J \Theta$ to obtain $T_{1}^{n}=Q_{1} U^{n} J_{1}, n \geq 0$.

We notice the following identity,

$$
J_{1} S=\left(S \oplus\left(I_{L^{p}\left(\Omega_{0}\right)} \otimes S\right)\right) J_{1},
$$


where $S: X \rightarrow X$ is a bounded operator which commutes with $T_{1}$. As, for $x_{0} \in \operatorname{Ker}\left(I_{X}-\right.$ $\left.T_{1}\right)$, and $x_{1} \in \overline{\operatorname{Ran}\left(I_{X}-T_{1}\right)}$ we have,

$$
\begin{aligned}
& \left(S \oplus\left(I_{L^{p}\left(\Omega_{0}\right)} \otimes S\right)\right) J \Theta\left(x_{0} \oplus x_{1}\right) \\
= & \left(S \oplus\left(I_{L^{p}\left(\Omega_{0}\right)} \otimes S\right)\right) J\left(x_{0} \oplus\left(I_{X}+T_{1}\right) x_{1}\right) \\
= & \left.\left(S \oplus\left(I_{L^{p}\left(\Omega_{0}\right)} \otimes S\right)\right)\left(x_{0} \oplus \sum_{k=0}^{\infty} \epsilon_{k} \otimes T_{1}^{k}\left(I_{X}-T_{1}\right)^{k}\right)^{\frac{1}{2}}\left(I_{X}+T_{1}\right) x_{1}\right) \\
= & \left.S x_{0} \oplus \sum_{k=0}^{\infty} \epsilon_{k} \otimes T_{1}^{k}\left(I_{X}-T_{1}\right)^{k}\right)^{\frac{1}{2}}\left(I_{X}+T_{1}\right) S x_{1} \\
= & J_{1} S\left(x_{0} \oplus x_{1}\right) .
\end{aligned}
$$

Let $\left(T_{2}, \ldots, T_{m}\right)$ be commuting tuple of Ritt operators each of which admits a bounded $H^{\infty}$ functional calculus. Hence by induction hypothesis, there exists a measure space $\Omega^{\prime \prime}$, a commuting tuple of isometric isomorphisms $\left(U_{2}, \ldots, U_{m}\right)$ on $L^{p}\left(\Omega^{\prime \prime}, X\right)$ together with two bounded operators $Q_{2}: L^{p}\left(\Omega^{\prime \prime}, X\right) \rightarrow X$ and $J_{2}: X \rightarrow L^{p}\left(\Omega^{\prime \prime}, X\right)$ such that

$$
T_{2}^{i_{2}} \cdots T_{m}^{i_{m}}=Q_{2} U_{2}^{i_{1}} \cdots U_{m}^{i_{m}} J_{2}
$$

for all $i_{2}, \ldots, i_{m} \in \mathbb{N}_{0}$. We notice the following,

$$
\begin{aligned}
& T_{1}^{i_{1}} T_{2}^{i_{2}} \ldots T_{m}^{i_{m}} \\
= & Q_{1} U^{i_{1}} J_{1} T_{2}^{i_{2}} \cdots T_{m}^{i_{m}} \\
= & Q_{1} U^{i_{1}}\left(T_{2}^{i_{2}} \cdots T_{m}^{i_{m}} \oplus\left(I_{L^{p}\left(\Omega_{0}\right)} \otimes T_{2}^{i_{2}} \cdots T_{m}^{i_{m}}\right)\right) J_{1} \\
= & Q_{1} U^{i_{1}}\left(Q_{2} \oplus\left(I_{L^{p}\left(\Omega_{0}\right)}^{m} \otimes Q_{2}\right)\right)\left(\prod_{j=2}^{m}\left(U_{j}^{i_{j}} \oplus\left(I_{L^{p}\left(\Omega_{0}\right)} \otimes U_{j}^{i_{j}}\right)\right)\right) \\
& \left(J_{2} \oplus\left(I_{L^{p}\left(\Omega_{0}\right)} \otimes J_{2}\right)\right) J_{1} \\
= & \mathcal{Q}\left(I_{L^{p}\left(\Omega^{\prime \prime}, X\right)} \oplus\left(\mathbf{u}^{i_{1}} \otimes I_{L^{p}\left(\Omega^{\prime \prime}, X\right)}\right)\right)\left(\prod_{j=2}^{m}\left(U_{j}^{i_{j}} \oplus\left(I_{L^{p}\left(\Omega_{0}\right)} \otimes U_{j}^{i_{j}}\right)\right)\right) \mathcal{J},
\end{aligned}
$$

where $\mathcal{Q}=Q_{1}\left(Q_{2} \oplus\left(I_{L^{p}\left(\Omega_{0}\right)} \otimes Q_{2}\right)\right)$ and $\mathcal{J}=\left(J_{2} \oplus\left(I_{L^{p}\left(\Omega_{0}\right) \otimes J_{2}}\right)\right) J_{1}$.

If $X$ is an ordered Banach space, a closed subspace of an $L^{p}$-space, or an $\mathrm{SQ}_{p}$ space, one can get the result similarly.

\section{Proofs of Theorem (2.11) and Theorem (2.12)}

We will use method of transference for proving the implication $(i i) \Rightarrow(i)$ in Theorem (2.11).

Proof of Theorem (2.11). Form Theorem (2.7) we have $\mathbf{T}$ admits joint isometric loose dilation. As $T_{i}$ satisfies $H^{\infty}$-functional calculus for each $i=1, \ldots, n$ by [4] we have $T_{i}$ 's are $R$-Ritt. This proves $(1) \Rightarrow(2)$.

Assuming (2), one can deduce (3) by using a matrix-valued version of Coifmann-Weiss general transference principle (Theorem 5.2.1, [12]). (3) $\Rightarrow(4)$ is trivial. Now for the remaining we have each $T_{i}$ is $R$-Ritt and $p$-polynomially bounded by [4], $I-T_{i}$ has bounded $H^{\infty}\left(\Sigma_{\theta_{i}}\right)$-functional calculus for some $\theta_{i} \in(0, \pi)$. Again by Theorem (2.5), [4] and [13] we have the required result.

It is known that Ritt operators which are positive contractions admit bounded $H^{\infty}$-functional calculus [5], [23]. So, by applying Theorem (2.11) we have the following weak version of AkcogluSucheston dilation theorem. 
Corollary 3.3. Let $\mathbf{T}=\left(T_{1}, \ldots, T_{n}\right)$ be an $n$-tuple of commuting Ritt operators which are also positive contraction on an $L^{p}$-space, $1<p<\infty$. Then $\mathbf{T}$ admits a joint isometric loose dilation.

Proof of Theorem (2.12). If each $T_{i}$ is similar to contraction then by [21] (Theorem 8.1) each $T_{i}$ admits a bounded $H^{\infty}$-functional calculus. Thus, (2) follows by [13]. Assuming (2), by Theorem (2.7), $\mathbf{T}$ has a joint isometric loose dilation on $L^{2}(\Omega, \mathcal{H})$ for some measure space $\Omega$. Therefore, $(2) \Longrightarrow(3)$ by [25] (Corollary 5.2). (3) $\Longrightarrow(1)$ is trivial.

Acknowledgement: We are thankful to Prof. C. Le Merdy for exposing us to the theory of Ritt operators and suggesting the problem as well as many valuable discussions and suggestions during this work. We appreciate Sayantan Chakrabarty's help in drawing the pictures.

\section{REFERENCES}

[1] Akcoglu, M. A. ; Sucheston, L. Dilations of positive contractions on $L_{p}$ spaces. Canad. Math. Bull. 20 (1977), no. 3, 285-292.

[2] Albrecht, D. W. Functional calculi of commuting unbounded operators. Diss. Monash University, 1994.

[3] Ando, T. On a pair of commutative contractions. Acta Sci. Math. (Szeged) 241963 88-90.

[4] Arhancet, C. ; Le Merdy, C. Dilation of Ritt operators on $L^{p}$-spaces. Israel J. Math. 201 (2014), no. 1, $373-414$.

[5] Arhancet, C. ; Fackler, S. ; Le Merdy, C. Isometric dilations and $H^{\infty}$ calculus for bounded analytic semigroups and Ritt operators. Trans. Amer. Math. Soc. 369 (2017), no. 10, 6899-6933.

[6] Bourgain, J. Vector-valued singular integrals and the $H^{1}-B M O$ duality.Probability theory and harmonic analysis (Cleveland, Ohio, 1983), 1-19, Monogr. Textbooks Pure Appl. Math., 98, Dekker, New York, 1986.

[7] Coifman, R. R. ; Weiss, G. Transference methods in analysis. Conference Board of the Mathematical Sciences Regional Conference Series in Mathematics, No. 31. American Mathematical Society, Providence, R.I., 1976. ii $+59 \mathrm{pp}$.

[8] Coifman, R. R. ; Richard, R. ; Guido, W. Applications of transference: the $L^{p}$ version of von Neumann's inequality and the Littlewood-Paley-Stein theory. Linear spaces and approximation (Proc. Conf., Math. Res. Inst., Oberwolfach, 1977), pp. 53-67.

[9] Cowling, M. ; doust, I. ; McIntosh, A.; Yagi, A. Banach space operators with a bounded $H^{\infty}$ functional calculus. J. Austral. Math. Soc. Ser. A 60 (1996), no. 1, 51-89

[10] Delaubenfels, R. Similarity to a contraction, for power-bounded operators with finite peripheral spectrum. Trans. Amer. Math. Soc. 350 (1998), no. 8, 3169-3191.

[11] Dore, G. ; and Alberto, V. $H^{\infty}$ functional calculus for sectorial and bisectorial operators. Studia Math. 166 (2005), no. 3, 221-241.

[12] Fendler, G. Dilations of one parameter semigroups of positive contractions on $L^{p}$ spaces. Canad. J. Math. 49 (1997), no. 4, 736-748.

[13] Franks, E. ; McIntosh, A. Discrete quadratic estimates and holomorphic functional calculi in Banach spaces. Bull. Austral. Math. Soc. 58 (1998), no. 2, 271-290.

[14] Gupta, R. ; Ray, S. K. On a Question of N. Th. Varopoulos. arXiv preprint arXiv:1611.06726 (2016). ( To appear in Annales de l'Institut Fourier).

[15] Halmos, P. R. Ten problems in Hilbert space. . Bull. Amer. Math. Soc. 761970 887-933.

[16] Kalton, N. J. ; Kucherenko. T. Sectorial operators and interpolation theory, 111-119, Contemp. Math., 445, Amer. Math. Soc., Providence, RI, 2007.

[17] Kalton, N. J. ; Weis. L. The $H^{\infty}$ calculus and sums of closed operators. Math. Ann. 321 (2001), no. 2, 319-345.

[18] Krengel, U. Ergodic theorems. With a supplement by Antoine Brunel. De Gruyter Studies in Mathematics, 6. Walter de Gruyter \& Co., Berlin, 1985. viii+357 pp.

[19] Lancien, F. ; Le Merdy C. On functional calculus properties of Ritt operators. Proc. Roy. Soc. Edinburgh Sect. A 145 (2015), no. 6, 1239-1250.

[20] Lancien, F. ; Gilles, L.; Le Merdy, C. A joint functional calculus for sectorial operators with commuting resolvents. Proc. London Math. Soc. (3) 77 (1998), no. 2, 387-414.

[21] Le Merdy, C. $H^{\infty}$ functional calculus and square function estimates for Ritt operators. Rev. Mat. Iberoam. 30 (2014), no. 4, 1149-1190.

[22] Le Merdy, C. The similarity problem for bounded analytic semigroups on Hilbert space. Semigroup Forum. Vol. 56. No. 2. Springer-Verlag, 1998.

[23] Le Merdy, C. ; Xu, Q. Maximal theorems and square functions for analytic operators on $L_{p}$-spaces. J. Lond. Math. Soc. (2) 86 (2012), no. 2, 343-365. 
[24] McIntosh, A. Operators which have an $H_{\infty}$ functional calculus. Miniconference on operator theory and partial differential equations (North Ryde, 1986), 210-231, Proc. Centre Math. Anal. Austral. Nat. Univ., 14, Austral. Nat. Univ., Canberra, 1986.

[25] Paulsen, V. Completely bounded maps and operator algebras. Cambridge Studies in Advanced Mathematics, 78. Cambridge University Press, Cambridge, 2002. xii+300 pp.

[26] Paulsen, V. Every completely polynomially bounded operator is similar to a contraction. J. Funct. Anal. 55 (1984), no. 1, 1-17.

[27] Peller, V. V. An Analogue of an inequality of J. von Neumann, isometric dilation of contractions, and approximation by isometries in spaces of measurable functions. (Russian) Spectral theory of functions and operators, II. Trudy Mat. Inst. Steklov. 155 (1981), 103-150, 185.

[28] Petrović, S. Polynomially unbounded product of two polynomially bounded operators. Integral Equations Operator Theory 27 (1997), no. 4, 473-477.

[29] Pisier, G. Similarity problems and completely bounded maps. Second, expanded edition. Includes the solution to "The Halmos problem". Lecture Notes in Mathematics, 1618. Springer-Verlag, Berlin, 2001. viii+198 pp.

[30] Pisier, G. A polynomially bounded operator on Hilbert space which is not similar to a contraction. J. Amer. Math. Soc. 10 (1997), no. 2, 351-369.

[31] Pisier, G. Joint similarity problems and the generation of operator algebras with bounded length. Integral Equations Operator Theory 31 (1998), no. 3, 353-370.

[32] Ray, S.K. On Multivariate Matsaev's Conjecture. arXiv preprint arXiv:1703.00733 (2017).

[33] Ray, S.K. On Functional Calculus For n-Ritt operators. arXiv preprint arXiv:1706.05856 (2017).

[34] Sz.-Nagy, B. ; Foias, C. Harmonic Analysis of Operators on Hilbert Spaces.Translated from the French and revised North-Holland Publishing Co., Amsterdam-London; American Elsevier Publishing Co., Inc., New York; Akadémiai Kiadó, Budapest 1970 xiii+389 pp.

[35] Varopoulos, N. T. On an inequality of von Neumann and an application of the metric theory of tensor products to operators theory. J. Functional Analysis 16 (1974), 83-100.

[36] von Neumann, J. Eine Spektraltheorie für allgemeine Operatoren eines unitären Raumes. Math. Nachr. 4, (1951). 258-281.

[37] Weis, L. The $H^{\infty}$ holomorphic functional calculus for sectorial operators-a survey. Partial differential equations and functional analysis, 263-294, Oper. Theory Adv. Appl., 168, Birkhäuser, Basel, 2006.

[38] Weis, L. Operator-valued Fourier multiplier theorems and maximal $L_{p}$-regularity. Math. Ann. 319 (2001), no. $4,735-758$.

Parasar Mohanty: Department of Mathematics and Statistics, Indian Institute of Technology, KANPUR-208016

Email address: parasar@math.iitk.ac.in

Samya Kumar Ray: Department of Mathematics and Statistics, Indian Institute of Technology, KANPUR-208016

Email address: samya@math.iitk.ac.in 